\title{
Synchronous nasopharyngeal carcinoma and cardia adenocarcinoma accompanied with suspected dermatomyositis: A case report and literature review
}

\author{
RUI AO ${ }^{1,2}$, YUHUI WANG ${ }^{1}$, DANDAN DONG $^{3}, \mathrm{KE} \mathrm{XIE}^{2}$ and ZHENGRONG WANG ${ }^{1}$ \\ ${ }^{1}$ Key Laboratory of Chronobiology of Health Ministry, Basic and Forensic School, Sichuan University; \\ Departments of ${ }^{2}$ Oncology and ${ }^{3}$ Pathology, Sichuan Academy of Medical Sciences, \\ Sichuan Provincial People's Hospital, Chengdu, Sichuan 610041, P.R. China
}

Received July 25, 2012; Accepted October 5, 2012

DOI: $10.3892 / 01.2012 .964$

\begin{abstract}
Synchronous multiple primary cancers are relatively uncommon and synchronous multiple cancers in coexistence with dermatomyositis (DM) are extremely rare. In the present study, we report a case of a 64-year-old male with nasopharyngeal undifferentiated non-keratinizing carcinoma and cardia adenocarcinoma which were diagnosed synchronously. In addition, suspected DM was diagnosed during the course of chemotherapy. The symptoms of DM were exaggerated even when methylprednisolone while administered. The patient succumbed to nutritional deterioration four months following his initial admission. In the present study, the etiologies of multiple primary cancers are discussed and a brief review of the literature on the correlation between malignance and DM was conducted.
\end{abstract}

\section{Introduction}

Multiple primary cancers are relatively uncommon. The present study reports a case in which synchronous nasopharyngeal undifferentiated non-keratinizing carcinoma and cardia adenocarcinoma were diagnosed. During the therapy course, progressive painless symmetric proximal muscle weakness and a skin rash emerged and were accompanied with dysphasia. In addition, suspected dermatomyositis (DM) was diagnosed. DM developed progressively in spite of application of methylprednisolone until the patient succumbed to nutritional deterioration on May 6, 2012. We present this case with two noteworthy aspects. Firstly, synchronous nasopharyngeal undifferentiated non-keratinizing carcinoma and cardia adenocarcinoma are relatively uncommon; and secondly the

Correspondence to: Dr Rui Ao, Key Laboratory of Chronobiology of Health Ministry, Basic and Forensic School, Sichuan University, Daxue Road, Chengdu, Sichuan 610041, P.R. China

E-mail: aorui1040@hotmail.com

Key words: synchronous multiple primary cancers, dermatomyositis coexistance of multiple primary cancers with DM is extremely rare and indicative of poor prognosis.

\section{Case reports}

Clinical presentation. The patient was a 64-year-old male from Sichuan, China. Prior to admittance in January 2012, the patient had experienced tinnitus of the right ear for one year and bloody nasal mucus for two months. A tumor was identified on the right and top wall of the nasopharynx by magnetic resonance imaging (MRI) and nasopharyngeal mirror. MRI also revealed that the right pharynx was absent and the right base of the skull had been destroyed by the tumor. Pathological results of the tumor confirmed that it was nasopharyngeal undifferentiated non-keratinizing carcinoma. Two hard lymph nodes in the right neck, with an approximate diameter of $2 \mathrm{~cm}$, were identified by physical examination. Comprehensive examination of the patient was performed. Abdominal computer tomography (CT) revealed multiple nodules in the liver, indicative of metastasized lesions. The diameter of these nodules ranged between 2 and $3 \mathrm{~cm}$. In addition, CT revealed a thicker than normal cardia tube wall. Gastroscopy was performed and a large neoplasm was identified surrounding almost the entire diameter of the cardia (Fig. 1). Pathological analyses were consistent with the diagnosis of cardia adenocarcinoma. In situ Epstein-Barr virus (EBV)-encoded small RNA (EBER) detection demonstrated that the nasopharyngeal carcinoma cells were positive and cardia carcinoma cells were negative for EBER (Fig. 2). The study was approved by the ethics committee of Sichuan Provincial People's Hospital, Chengdu, China, and written consent was obtained from the patient's family

Treatment and clinical course. The patient received 2 cycles of chemotherapy as follows: lipid body paclitaxel was administered at a dose of $210 \mathrm{mg}$ on day 1; nedaplatin was administered at a dose of $50 \mathrm{mg}$ on day 1,2 and 3. Courses were repeated every 21 days. MRI and CT scans were repeated to evaluate the effects of chemotherapy. MRI demonstrated that the nasopharyngeal tumor had shrunk and CT revealed that the nodules of liver and tumor of cardia remained 


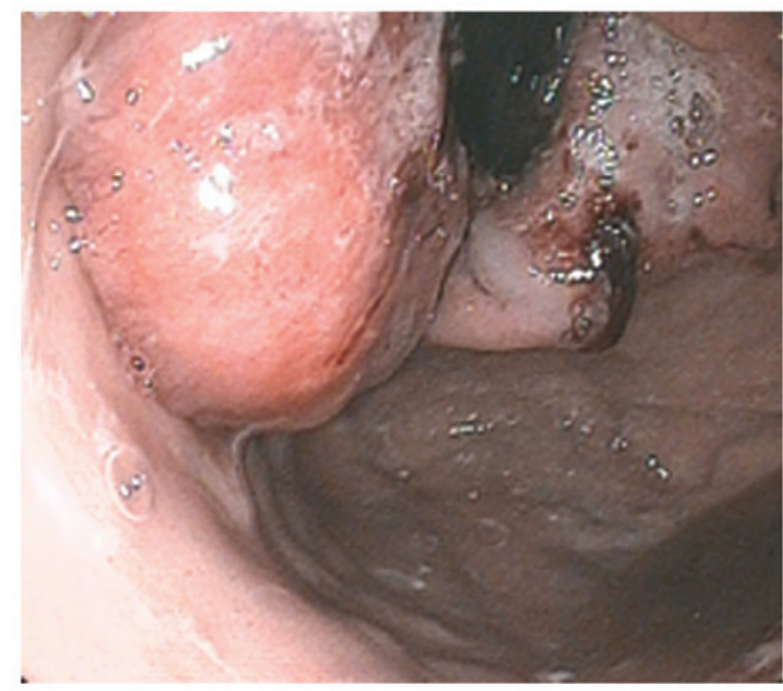

Figure 1. Cardia tumor as examined by gastroscopy.
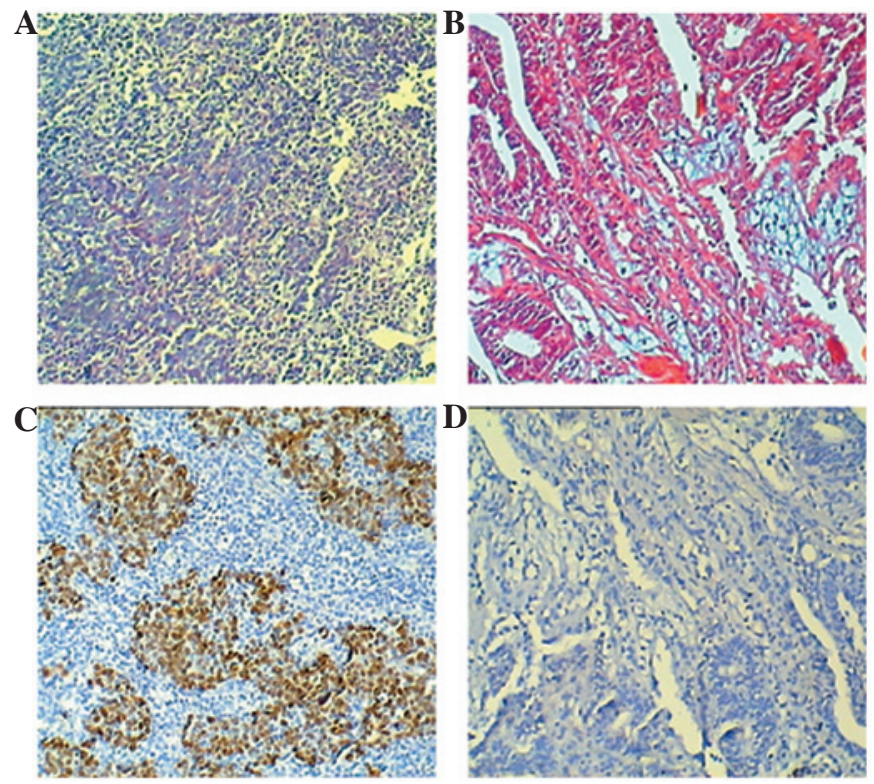

Figure 2. Results of histopathology and EBER in situ hybridization of nasopharyngeal carcinoma and cardia adenocarcinoma. (A) Nasopharyngeal undifferentiated non-keratinizing carcinoma (HE stain; magnification, x100). (B) Cardia adenocarcinoma (HE stain; magnification, x100). (C) EBER in situ hybridization of nasopharyngeal carcinoma. (D) EBER in situ hybridization of cardia adenocarcinoma.

stable (Fig. 3). Following two cycles of chemotherapy, rashes were identified on the back of the neck of the patient. Rashes gradually diffused to the hands and face and formed erythema with dander (Fig. 4). Specific anti-allergy drugs were administered to the patient but had limited effect on the erythema. During the third cycle of chemotherapy, proximal limb muscle strength of the patient decreased without pain and presented as difficulty in climbing steps, rising from a chair, lifting objects and combing hair. This observation was also accompanied by dysphasia. Serum creatine kinase levels were analyzed and detected at $214 \mathrm{U} / 1$ (normal value, 26-174 U/1). A previous study by Bohan and Peter (1) reported suspected dermatomyositis, however, muscle biopsy was refused by the patient
A

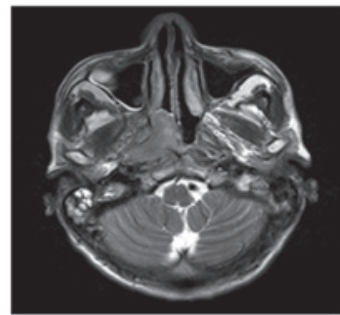

$\mathbf{B}$

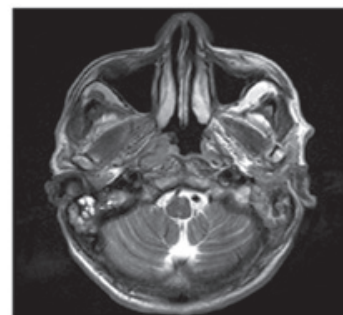

C

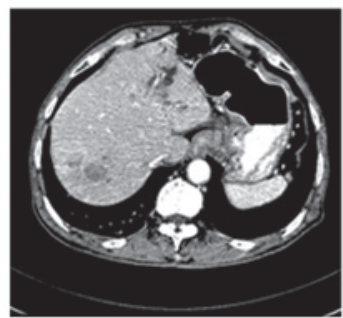

D

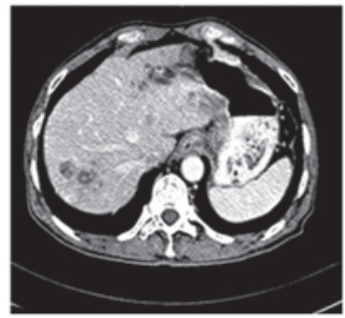

Figure 3. Nasopharyngeal MRI and abdominal CT prior to and following chemotherapy. (A) Nasopharyngeal MRI prior to chemotherapy. (B) Nasopharyngeal MRI following chemotherapy revealing that the tumor had reduced in size. (C) Abdominal CT prior to chemotherapy demonstrating a thicker cardia tube wall than normal. Metastatic nodules of liver are also revealed (D) Abdominal CT following chemotherapy.
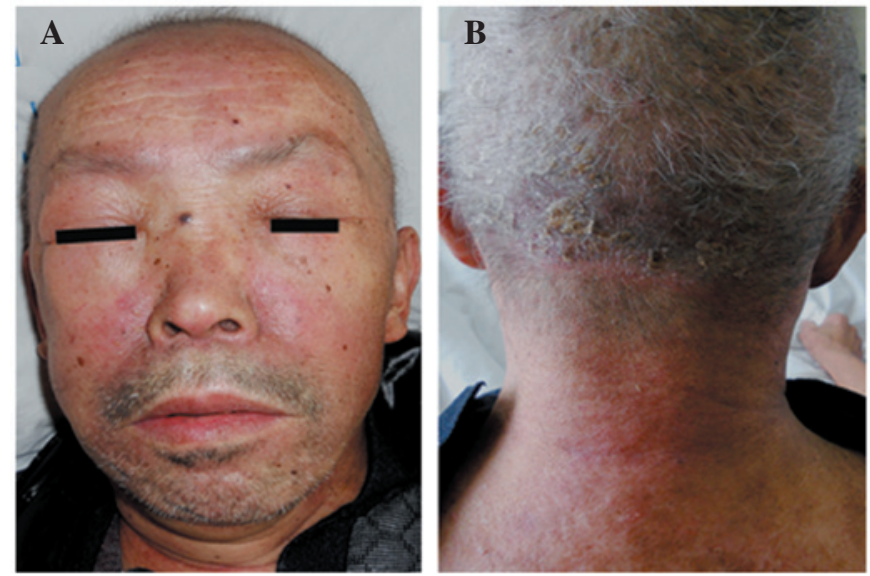

Figure 4. Erythema, edema and dander on the head and neck. (A) Front; (B) back.

and result of electromyography test was normal. The present patient received intravenous infusion of methylprednisolone $40 \mathrm{mg} /$ day but limited alleviation of symptoms was observed. The characteristic symptoms of dermatomyositis advanced and the patient deteriorated rapidly and succumbed to nutritional deterioration on May 6, 2012.

\section{Discussion}

Multiple primary cancers are relatively uncommon. In a Japanese large-scale survey, approximately $4.2 \%$ of patients were diagnosed with synchronous multiple primary cancers upon initial admission to the hospital (2). Analysis of the etiology of multiple primary cancers is important for understanding a number of factors, including genetic susceptibility and common exposure status (exposure to an agent carcinogenic to both sites) and may prove valuable in tumor prevention and treatment. Patients with Li-Fraumeni syndrome (a domi- 
nantly inherited disorder) or a history of cigarette and alcohol consumption are associated with a high risk of developing multiple primary cancers $(3,4)$. EBV is a member of the herpes virus family. Currently, three EBV nuclear antigens are associated with cancer development, EBNA-1 and -3C and LMP-1. These antigens promote genomic instability, increase the rate of somatic hypermutations and obstruct apoptotic pathways. Nasopharyngeal non-keratinizing carcinomas are markedly associated with EBV. In addition, EBV is correlated with a number of other types of cancer, including Burkitt's, Hodgkin's and non-Hodgkin's lymphoma, gastric carcinoma and leiomyosarcomas. A previous study reported detection of EBV in more than $90 \%$ of gastric lymphoepithelioma-like carcinomas and $5-25 \%$ of gastric adenocarcinomas (5). Following synchronous nasopharyngeal carcinoma and cardia adenocarcinoma diagnosis of the present patient, a hypothesis emerged associating the two tumors with EBV. However, results of EBER detection were not consistent with this hypothesis. The nasopharyngeal non-keratinizing carcinoma was identified as EBV-positive, however, the cardia adenocarcinoma was negative for EBV. Results of the literature review revealed that EBV-associated multiple primary cancers (defined as the positive detection of EBV in all primary cancers) is extremely rare. Wang et al analysed the correlation between the ratio of second primary tumors with nasopharyngeal carcinoma and EBV. The group identified that gastric cancer was the second leading second primary tumor in nasopharyngeal non-keratinizing carcinoma patients. However, the results also demonstrated that only $1 / 11$ second primary gastric cancer specimens were EBV-positive and an increased detection of EBV was not reported (6). The majority of adult individuals are infected with EBV (95\%), however, only a small number of these cases develop into malignances. The distribution of EBV-associated cancers is geography defined. EBV-related Burkitt's lymphoma occurs primarily in regions of equatorial Africa. Nasopharyngeal carcinoma is rare in most parts of the world, however, it is prevalent in South China, indicative of additional factors important for the development of specific types of tumors. Although EBV infection is correlated with a number of tumor types, it is not currently considered to be sufficient for the induction of cancer alone, instead it appears to promote the induction of tumorigenesis by additional oncogenic factors.

DM is an autoimmune disorder characterized by inflammatory muscular and cutaneous disease. Individuals with DM have a high risk of developing a malignancy. It is currently estimated that 8-60\% of individuals with DM also have cancer (7). Malignancies have been identified prior to, following and in tandem with DM diagnosis and are most commonly identified one year following diagnosis. Cross-immune responses against myositis autoantigens or viral infections, including $\mathrm{EBV}$, are thought to be responsible for the correlation between malignancy and DM $(8,9)$. Common malignancies identified in individuals with DM include adenocarcinoma of the ovary, lung or gastrointestinal tract in western countries and nasopharyngeal non-keratinizing carcinoma in South Asia and China. Individuals with these correlations commonly present with skin lesions and muscle weakness. Patients presenting with DM and a malignancy are not sensitive to glucocorticoid, however, the symptoms of DM would be alleviated accompanied by remission of the tumor after antitumor therapy in the majority of patients (10). The current patient exhibited two primary synchronous tumours and did not respond to chemotherapy or corticosteroids, indicative of a more complex disorder involving the immune system and poor prognosis.

\section{References}

1. Bohan A and Peter JB: Polymyositis and dermatomyositis (first of two parts). N Engl J Med 292: 344-347, 1975.

2. Kaneko S and Yamaguchi N: Epidemiological analysis of site relationships of synchronous and metachronous multiple primary cancers in the National Cancer Center, Japan, 1962-1996. Jpn J Clin Oncol 29: 96-105, 1999.

3. Hisada M, Garber JE, Fung CY, Fraumeni JF Jr and Li FP: Multiple primary cancers in families with Li-Fraumeni syndrome. J Natl Cancer Inst 90: 606-611, 1998.

4. Wynder EL, Mushinski MH and Spivak JC: Tobacco and alcohol consumption in relation to the development of multiple primary cancers. Cancer 40: 1872-1878, 1977.

5. Young LS and Murray PG: Epstein-Barr virus and oncogenesis: from latent genes to tumours. Oncogene 22: 5108-5121, 2003.

6. Wang CC, Chen ML, Hsu KH, et al: Second malignant tumors in patients with nasopharyngeal carcinoma and their association with Epstein-Barr virus. Int J Cancer 87: 228-231, 2000.

7. Zahr ZA and Baer AN: Malignancy in myositis. Curr Rheumatol Rep 13: 208-215, 2011

8. Casciola-Rosen L, Nagaraju K, Plotz P, et al: Enhanced autoantigen expression in regenerating muscle cells in idiopathic inflammatory myopathy. J Exp Med 201: 591-601, 2005.

9. Chen DY, Chen YM, Lan JL, et al: Polymyositis/dermatomyositis and nasopharyngeal carcinoma: the Epstein-Barr virus connection? J Clin Virol 49: 290-295, 2010

10. Pectasides D, Koumpou M, Gaglia A, et al: Dermatomyositis associated with breast cancer. Anticancer Res 26: 2329-2331, 2006. 